\title{
Asociación entre el tiempo de exposición a pantallas y rendimiento en una prueba de tamizaje de desarrollo infantil
}

\author{
Association between screen time and children's performance on a developmental screening test
}

\section{Comentado de:}

Madigan S et al. JAMA Pediatrics. 2019. PMID: $30907942^{1}$

\section{Objetivo}

Evaluar en qué dirección se da la potencial asociación causal entre el tiempo de exposición a las pantallas y el neurodesarrollo infantil en una población de madres y niñas/os.

\section{Diseño, población y medición de resultados principa- les}

Estudio de cohorte de 2.441 díadas madre-hija/o canadienses, extraída del estudio All Our Families ${ }^{2,3}$. Los datos fueron recolectados entre 2011 y 2016 . Se utilizó un modelo de análisis estadístico en el cual se evaluaron datos longitudinales a través de mediciones repetidas en los meses 24,36 y 60 sobre exposición a pantallas (horas/semana) y puntajes de una prueba de tamizaje de neurodesarrollo (ASQ-3). Todos los datos fueron reportados por las madres. El modelo utilizado evaluó las relaciones recíprocas a lo largo del tiempo, procurando revelar la existencia de una relación causa-efecto.

\section{Resultados principales}

La mayor exposición a las pantallas (potencial causa) durante los meses 24 y 36 se asoció en forma significativa con menores puntajes en la prueba de tamizaje de neurodesarrollo infantil (potencial efecto) en los meses $36(\beta-0,06$; Intervalo de Confianza [IC] $95 \%-0,10$ a $-0,01)$ y $60(\beta-0,08 ;$ IC95\% $-0,13$ a $-0,02)$ respectivamente.

Por el contrario, cuando se realizó un análisis en la dirección inversa, no se observó una asociación estadísticamente significativa entre menores puntajes en el neurodesarrollo infantil (potencial causa) y un mayor tiempo posterior de exposición a las pantallas (potencial efecto).

\section{Conclusiones}

Los resultados apoyan la asociación direccional entre el tiempo de exposición a las pantallas y el desarrollo infantil. Comprender esta direccionalidad permite comprometer a las familias a hacer un uso racional y positivo de las tecnologías, para evitar las potenciales consecuencias derivadas de un exceso de su uso.

Fuente de financiamiento: Alberta Innovates Health Solutions Interdisciplinary Team.

\section{Comentario}

Diferentes sociedades y asociaciones pediátricas han desarrollado recomendaciones ${ }^{4,5}$ sobre el uso de pantallas en niños basadas en estudios primarios ${ }^{6-12}$, con excepción de la Sociedad Canadiense de Pediatría, que basa sus recomendaciones en una revisión sistemática ${ }^{13}$ llevada a cabo por la Sociedad Canadiense por la Fisiología del Ejercicio. La gran mayoría coincide en limitar el tiempo de exposición a pantallas a no más de una hora por día en el grupo etario de dos a cinco años. Estos estudios primarios, realizados en poblaciones de niños de seis meses a diez años de edad, habían documentado la asociación entre el mayor tiempo de exposición a pantallas y un peor desarrollo cognitivo, del lenguaje y motor, así como también un peor rendimiento escolar. También han encontrado asociación entre la exposición a contenido dirigido a adultos y peores resultados.

Este estudio es el primero en demostrar la direccionalidad de estas asociaciones encontradas en dichos estudios, a través de un diseño acorde a la pregunta planteada por los investigadores. Dado que se trató de un estudio longitudinal, fueron consideradas como variables independientes o explicativas (potencialmente causales) los tiempos anteriores de la cohorte. En este tipo de estudios, dichos coeficientes tienen un fuerte peso ya que permiten explicar lo que sucede en un momento dado (puntaje neurocognitivo) por lo sucedido previamente (puntaje neurocognitivo inmediatamente anterior).

Entre las limitaciones para interpretar sus resultados, vale destacar que desde que fue recolectada la información sobre la que fue realizado este trabajo (2011 y 2016) pueden haberse modificado los estímulos que reciben los niños expuestos a las pantallas (p. ej. en el nivel de proactividad/pasividad que estos motivan) y los comportamientos de los niños frente a estas (más o menos pasivos). Por otro lado, la primera evaluación de la investigación que hemos resumido fue realizada a los 24 meses de edad, mientras que investigaciones recientes ${ }^{14}$ indican que la exposición a las pantallas se está dando cada vez a edades más tempranas, con lo cual podría resultar beneficioso incluir datos adicionales a los 12 ó 18 meses para brindar mayor apoyo (o cuestionar) los patrones observados en este estudio.

En este tipo de investigaciones sería importante implementar un enfoque causal multidimensional, que incluya otras variables, como el contenido de la exposición a las pantallas (no sólo el tiempo), la supervisión por parte de adultos, el tipo de pantalla utilizada, etc.

Por otro lado, más allá de que suele argumentarse que el tiempo invertido en pantallas es tiempo que se le resta a oportunidades valiosas para practicar y dominar habilidades interpersonales, motrices y de comunicación, quedan dudas si el método utilizado para evaluar dichas habilidades pudo haber quedado obsoleto. Y si bien suele aconsejarse un uso supervisado de las pantallas y la selección de su contenido -con lo cual coincido-, me atrevo a poner en duda si la limitación en el tiempo debiera ser tan estricta como lo plasman las asociaciones y sociedades de pediatría en sus guías, teniendo en cuenta que la asociación con evidencia más fuerte al día de hoy de tiempo de pantallas es un mayor riesgo de desarrollo de obesidad y síntomas depresivos ${ }^{15}$. 
Parece más sensato en este sentido, lo que plantea la guía del Colegio Real de Pediatría del Reino Unido ${ }^{16}$ : brindar información basada en la evidencia y ayudar a cada familiar a que "diagnostique" su situación con respecto a las pantallas y tome las medidas que considere apropiadas.

\section{Conclusiones de la comentadora}

El diseño de este estudio es apropiado para demostrar una relación causal a través de la documentación de efectos diferidos en el tiempo (a mayor exposición, peor rendimiento en las pruebas de tamizaje del desarrollo psicomotriz). Sin embargo, sus resultados deberían leerse en forma crítica teniendo en cuenta el mundo digitalizado en el que vivimos y del que difícilmente podamos abstraernos.

Leticia Gavoto [ Servicio de Medicina Familiar y Comunitaria, Hospital Italiano de Buenos Aires, CABA, Ciudad Autónoma de Buenos Aires, Argentina. leticia.gavoto@hospitalitaliano.org.ar ]

Gavoto L Asociación entre el tiempo de exposición a pantallas y rendimiento en una prueba de tamizaje de desarrollo infantil. Evid Actual Pract Ambul. 2020;23(1):e002025. Comentado de: Madigan S, Browne D, Racine N, Mori C, Tough S. Association Between Screen Time and Children's Performance on a Developmental Screening Test [published correction appears in JAMA Pediatr. 2019 May 1;173(5):501-502]. JAMA Pediatr. 2019;173(3):244-250. PMID: 30688984

\section{Referencias}

1. Madigan S, Browne D, Racine N, et al. Association between screen time and children's performance on a developmental screening test. JAMA Pediatrics. 2019;173(3):244-244. Available from: 10.1001/jamapediatrics.2018.5056;https://dx.doi.org/10.1001/jamapediatrics.2018.5056.

2. Tough SC, McDonald SW, Collisson BA, et al. Cohort profile: the All Our Babies pregnancy cohort (AOB). International Journal of Epidemiology. 2017;46(5):1389-1390k. Available from: 10.1093/ije/dyw363;https://dx.doi.org/10.1093/ije/dyw363.

3. McDonald SW, Lyon AW, Benzies KM, et al. The All Our Babies pregnancy cohort: design, methods, and participant characteristics. BMC Pregnancy and Childbirth. 2013;13(Suppl 1):S2-S2. Available from: 10.1186/1471-2393-13-s1-s2; https://dx.doi.org/10.1186/1471-2393-13-s1-s2.

4. Council on Communications and Media. Media and young minds. Pediatrics. 2016;138(5):e20162591. Available from: 10.1542/peds.2016-2591.

5. Subcomisión de Tecnologías de Información y Comunicación. Bebés, niños, adolescentes y pantallas: ¿qué hay de nuevo? Arch Argent Pediatr. 2017;115(4):404-408. Available from: 10.5546/aap.2017.404; https://www.sap.org.ar/docs/publicaciones/archivosarg/2017/v115n4a31.pdf.

6. Tomopoulos S, Dreyer BP, Berkule S, et al. Infant media exposure and toddler development. Archives of Pediatrics \& Adolescent Medicine. 2010;164(12):1105-1116. Available from: 10.1001/archpediatrics.2010.235; https://dx.doi.org/10.1001/archpediatrics.2010.235.

7. Lin LY, Cherng RJ, Chen YJ, et al. Effects of television exposure on developmental skills among young children. Infant Behav Dev. $2015 ; 38: 20-26$. Available from: 10.1016/j.infbeh.2014.12.005

8. Zimmerman FJ, Christakis DA, Meltzoff AN. Associations between media viewing and language development in children under age 2 years. The Journal of Pediatrics. 2007;151(4):364-368. Available from: 10.1016/j.jpeds.2007.04.071;https://dx.doi.org/10.1016/j.jpeds.2007.04.071.

9. Duch H, Fisher EM, Ensari I, et al. Association of screen time use and language development in hispanic toddlers. Clinical Pediatrics. 2013;52(9):857865. Available from: 10.1177/0009922813492881; https://dx.doi.org/10.1177/0009922813492881.

10. Pagani LS, Fitzpatrick C, Barnett TA, et al. Prospective associations between early childhood television exposure and academic, psychosocial, and physical well-being by middle childhood. Archives of Pediatrics \& Adolescent Medicine. 2010;164(5):425-431. Available from: 10.1001/archpediatrics. 2010.50; https://dx.doi.org/10.1001/archpediatrics.2010.50.

11. Christakis DA, Gilkerson J, Richards JA, et al. Audible television and decreased adult words, infant vocalizations, and conversational turns. Archives of Pediatrics \& Adolescent Medicine. 2009;163(6):554-554. Available from: 10.1001/archpediatrics.2009.61;https://dx.doi.org/10.1001/archpediatrics. 2009.61.

12. Nathanson Al, Aladé F, Sharp ML, et al. The relation between television exposure and executive function among preschoolers. Developmental psychology. 2014;50(5):1497-1506. Available from: 10.1037/a0035714;https://dx.doi.org/10.1037/a0035714.

13. Tremblay MS, Chaput JP, Adamo KB, et al. Canadian 24-hour movement guidelines for the early years (0-4 years): an integration of physical activity, sedentary behaviour, and sleep. BMC Public Health. 2017;17(5):874-874. Available from: 10.1186/s12889-017-4859-6.

14. Kılıc AO, Sari E, Yucel H, et al. Exposure to and use of mobile devices in children aged 1-60 months. European Journal of Pediatrics. 2019;178(2):221-227. Available from: 10.1007/s00431-018-3284-x;https://dx.doi.org/10.1007/s00431-018-3284-x.

15. Stiglic N, Viner RM. Effects of screentime on the health and well-being of children and adolescents: a systematic review of reviews. BMJ Open. 2019;9(1):e023191-e023191. Available from: 10.1136/bmjopen-2018-023191;https://dx.doi.org/10.1136/bmjopen-2018-023191.

16. Royal College of Paediatrics and Child Health. The health impacts of screen time - a guide for clinicians and parents; 2019. Available from: https://www.rcpch.ac.uk/resources/health-impacts-screen-time-guide-clinicians-parents [Last access: 2020-03-27]. 